\title{
Protocol of Explanatory Study on Social, Economic and Political Dimensions of Nutritional Status of Children Aged Six to Sixty Months from Low Socio-Economic Strata in Kerala, India
}

\author{
Rajeev Jayalakshmi ${ }^{1,2 *}$, Srinivasan $\operatorname{Kannan}^{1}$ \\ ${ }^{1}$ Achutha Menon Centre for Health Science Studies, Sree Chitra Tirunal Institute for Medical \\ Sciences and Technology, Medical College PO, Thiruvananthapuram-695011, Kerala, India. \\ ${ }^{2}$ Department of Public Health and Community Medicine, Central University of Kerala, \\ Tejaswini Hills, Periye, Kasaragod-671316, Kerala, India
}

\begin{abstract}
Background: Despite having better human development indicators, one-fifth of under-five children in Kerala are undernourished. The demographic, socio-economic, cultural and political transition in Kerala necessitates a revisit on the causes of undernutrition among children given that it tackled many of the immediate causes of undernutrition. The present study aims at the multi-dimensional factors' influence on nutritional status of children from low socio-economic strata in Kerala.

Methods/Design: The conceptual framework for the present study adapted the ecosocial theory by Nancy Krieger. We use an explanatory study design, by adopting both quantitative and qualitative methods. The sample size for the quantitative phase is 600 children of age six to 60 months and their mothers. The qualitative phase includes in-depth interviews with key informants. The data collection tools include weighing scale and stadiometer for taking anthropometric measurements, household survey questionnaire, interview schedule for mothers, interview guide for key informants, field diary and checklist for document analysis.

Discussion: Contextual evidence that the study generates will have implications at individual, service provision and policy perspectives of undernutrition among children in Kerala. It will help improve the nutrition-specific and nutrition-sensitive policies and programs in Kerala, which in turn will improve the nutritional status of the children.
\end{abstract}

Keywords: nutritional status, under-five children, multidimensional causes, Kerala, India.

\section{Background}

Nutrition plays an important role in ensuring a child's survival and healthy growth to full potential in early childhood and productive adulthood. Nutrition imbalance can have both short and long-term repercussions on children reflected by growth failure, poor cognitive

\footnotetext{
* Correspondence to Rajeev Jayalakshmi, ${ }^{1}$ Achutha Menon Centre for Health Science Studies, Sree Chitra Tirunal Institute for Medical Sciences and Technology, Medical College PO, Thiruvananthapuram-695011, Kerala, India. Email: jayalakshmirajeev@cukerala.ac.in Social Science Protocols, February 2021, 1-12. http://dx.doi.org/10.7565/ssp.v4.5284
} 
development, frequent illnesses, poor school achievements etc. (United Nations Children's Fund, 2019).

According to the estimates of World Health Organization (WHO), around 5.2 million under-five children died in 2019; about $45 \%$ of those deaths were contributed by undernutrition directly or indirectly (WHO, 2020). Asia and Africa are the homes for most of the undernourished children of the world (United Nations Children's Fund [UNICEF], WHO, $\&$ World Bank, 2019). The prevalence of stunting, underweight and wasting among underfive children in India is far beyond the respective trigger levels of $20 \%, 15 \%$ and $5 \%$ given by the WHO for any population (WHO, 2010).

According to the fourth National Family Health Survey (NFHS-4), conducted in 2015-16, the prevalence of stunting, underweight and wasting among Indian children were $35.4 \%$, $35.7 \%$ and $21.0 \%$ respectively. Average annual reduction for all the three indicators since NFHS-3 (2005-06) was less than one per cent (International Institute for Population Sciences [IIPS] \& Inner City Fund [ICF], 2017a). In addition to this high burden, there is high interstate and intrastate disparities throughout the country that the majority of the undernourished children belong to the six states out of 29 states and seven union territories of India. The names of the states are Orissa, Chhattisgarh, Jharkhand, Assam, Uttar Pradesh and Rajasthan (Black et al., 2013). Despite the time to time nutrition-specific and/or nutritionsensitive interventions such as Integrated Child Development Services (ICDS), Public Distribution System (PDS) and Mahatma Gandhi National Rural Employment Guarantee Scheme that run across the country to ensure nutrition, food insecurity and minimum income respectively, the quest of undernutrition among children in the country could not be resolved (Planning Commission, 2010).

In terms of human development indicators, Kerala a southern state of India performed exceptionally compared to others. In Kerala, literacy rate, sex ratio, life expectancy, infant mortality rate and maternal mortality ratio etc. are far ahead of other states of India, and comparable with developed countries (Anand \& Sen, 2000; Devika, 2010). On the contrary, one in every five children in Kerala are undernourished and the annual average reduction of undernutrition is less than one percent during the last 25 years (IIPS \& ICF, 2017b). According to the NFHS-4 (2015-2016), the prevalence of stunting, underweight and wasting among under-five children in Kerala was $19.7 \%, 16.1 \%$ and $15.7 \%$ respectively (IIPS \& ICF, 2017b). Despite its achievements in stunting, the underweight and wasting among children are still higher than the WHO trigger levels (WHO, 2010). In Kerala, the poverty rate is less than 10 per cent and it might be reflecting in the reduced stunting levels (World Bank, 2017a). The alarmingly high levels of wasting (15.6\%) among children is a paradox to the achievement in economic status of the people and hence it is important to address this contrasting scenario of undernutrition among children in Kerala.

Table 1 well describes the intrastate disparities in undernutrition. Rural/urban disparities are also evident; undernutrition among children from urban areas is higher than that among children from rural areas (IIPS \& ICF, 2017b). 
Table 1 District-wise undernutrition among under-five children in Kerala, NFHS-4.

\begin{tabular}{|l|c|c|c|}
\hline \multicolumn{1}{|c|}{ District } & Stunting (Urban, rural) & Wasting (Urban, rural) & Underweight (Urban, rural) \\
\hline Alappuzha & $14.5(12.8,16.4)$ & $16.6(13.7,19.7)$ & $17.2(14.0,20.6)$ \\
\hline Ernakulam & $12.4(11.1,14.9)$ & $15.9(18.7,10.1)$ & $12(12.8,10.6)$ \\
\hline Idukki & 15.1 & 24.2 & 14.8 \\
\hline Kannur & $25.3(28.9,15.9)$ & $10.2(7.5,17.1)$ & $10.5(8.5,15.9)$ \\
\hline Kasaragod & $18.7(18.1,19.0)$ & $9.7(9.1,10.1)$ & $13.9(19.9,9.9)$ \\
\hline Kollam & $14.4(7.3,22.2)$ & $18.8(20.6,16.8)$ & $14.2(12.4,11.3)$ \\
\hline Kottayam & 22.0 & 16.2 & 11.3 \\
\hline Kozhikode & $18.0(19.3,15.6)$ & $13.5(10.1,19.9)$ & $18.5(18.5,18.9)$ \\
\hline Malappuram & $26.3(24.1,28.7)$ & $22.3(23.8,20.7)$ & $17.3(17.8,16.9)$ \\
\hline Palakkad & 20.2 & 10.3 & 19.1 \\
\hline Pathanamthitta & 13.3 & 14.4 & 11.4 \\
\hline Thiruvananthapuram & $19.5(24.3,14.5)$ & $13.1(11.5,14.8)$ & $21.6(19.8,23.3)$ \\
\hline Thrissur & $20.8(20.7,21.0)$ & $15.3(15.2,15.4)$ & $14(12.5,16.9)$ \\
\hline Wayanad & 27.7 & 23.9 & 27.2 \\
\hline Kerala & $19.7(19.8,19.5)$ & $15.7(16.0,15.5)$ & $16.1(15.5,16.7)$ \\
\hline
\end{tabular}

Source: International Institute for Population Sciences (IIPS) and Inner City Fund (ICF) International, 2017.

\subsection{Multifaceted causes of undernutrition}

The causes and effects of undernutrition are multifaceted. They are not mutually exclusive, instead closely linked to each other (Fenske, Burns, Hothorn, \& Rehfuess, 2013; Birhanu, 2015; Nisbett, Gillespie, Haddad, \& Harris, 2014; Wondimagegn, 2014). The three types of causes of undernutrition are immediate causes, underlying causes and basic causes according to the framework published by the United Nations Children's Fund (UNICEF) in 1990 (UNICEF, 2015). Researchers who studied various aspects of nutrition also tried to understand the phenomenon from multiple, interlinked points such as individual, household, community and policy levels (Chaturvedi et al., 2016; Kanter, Walls, Tak, Roberts, \& Waage, 2015; United States Dietary Guidelines Advisory Committee, 2010). The multiple dimensions of child nutrition identified through rigorous literature search are summarised in Table 2.

Studies across the world over the years have shown that the burden of undernutrition is skewed towards children from marginalized communities. Being children from rural areas and slums, poor economic strata, scheduled caste or tribal community etc. increase the vulnerability to be undernourished (Prabhakaran et al., 2013; Sabharwal, 2011; Van de Poel, Hosseinpoor, Speybroeck, Van Ourti, \& Vega, 2008). 
Table 2. Social, economic and political dimensions of nutritional status.

\begin{tabular}{lll}
\hline $\begin{array}{l}\text { Social } \\
\text { dimension }\end{array}$ & $\begin{array}{l}\text { Child } \\
\text { characteristics }\end{array}$ & $\begin{array}{l}\text { Age and sex of the child, birth weight, birth order, } \\
\text { breastfeeding \& weaning practices, immunization status, } \\
\text { acute or chronic illness }\end{array}$ \\
& $\begin{array}{l}\text { Family } \\
\text { Parental }\end{array}$ & $\begin{array}{l}\text { Type of family, family size } \\
\text { Parents' age, education and occupation; number of } \\
\text { children; mother's freedom of mobility and household } \\
\text { decision making, mother's awareness about nutrition }\end{array}$
\end{tabular}

Social Place of residence (urban/rural coastal), religion, caste, stratification experience of social isolation

\begin{tabular}{|c|c|c|}
\hline \multirow{2}{*}{$\begin{array}{l}\text { Economic } \\
\text { dimension }\end{array}$} & Household & Income, standard of living, consumption expenditure \\
\hline & Community & $\begin{array}{l}\text { Availability and access to food at the local level, price of } \\
\text { food and non-food items }\end{array}$ \\
\hline
\end{tabular}

The scenario of undernutrition among under-five children in Kerala is better compared to other states of the country since Kerala has tackled many of the immediate causes of undernutrition such as housing, access to safe water, hygiene and sanitation, immunization, mothers' education and age at marriage, health and other infrastructure and so on (Parayil, 1996; Ramachandran, 2000). In addition to the above, the programmes such as Integrated Child Development Services (ICDS), Public Distribution System (PDS), and Mahatma Gandhi National Rural Employment Guarantee Scheme (MGNREGS) are operational in the state to improve the nutritional status of children, to ensure household food security and to alleviate poverty in rural areas (Kerala State Planning Board, 2020). Even with this favouring environment, the progress of eliminating undernutrition among under-five children is unacceptably slow. It points towards the lacunae in the present system. Nevertheless, Kerala is a rapidly urbanizing state with a huge social, cultural and economic transition in the last few years. The rural/urban demarcation is disappearing at a faster rate (George \& Chattopadhyay, 2001). The state had the highest per capita income in the country and the highest consumption inequality as well (World Bank, 2017a) The poverty rate in rural areas of Kerala was $7.3 \%$ whereas in urban areas it was $15.3 \%$ (Kerala State Planning Board, 2019).

According to the World Bank's social inclusion data, the poverty rates among scheduled caste and scheduled tribes in Kerala were $16 \%$ and $39 \%$. These rates were three and six times higher compared to that of general population (World Bank, 2017b). The reports of higher prevalence of under-nutrition, and neonatal and infant deaths among tribal population of Kerala draws our attention to the social inequality, and inadequacy of the present system to address them (Philip et al., 2015; Prabhakaran et al., 2013; Sajithamohan \& Arunachalam, 2018).

\subsection{Objectives of the study}

1. To study the effect of socio-demographic and economic status on the nutritional status of children aged 6 to 60 months in Kerala.

2. To understand how the household food security affects the nutritional status of children aged 6 to 60 months from poor socio-economic strata in Kerala.

Social Science Protocols, February 2021, 1-12. 
3. To study the effect of social stratification on the nutritional status of children aged 6 to 60 months from poor socio-economic strata in Kerala.

a. To study the effect of social position (place of residence, religion, caste) on nutritional status of children aged 6 to 60 months from poor socio-economic strata in Kerala.

b. To understand the power structure within the community and its effect on the nutritional status of children aged 6 to 60 months from poor socio-economic strata in Kerala.

4. To understand the effect of policies on the nutritional status of children aged 6 to 60 months from poor socio-economic strata in Kerala.

a. To understand how ICDS contributes to the nutritional status of children aged 6 to 60 months from poor socio-economic strata in Kerala.

b. To study how the PDS contributed to household food security and its effect on the nutritional status of children aged 6 to 60 months from poor socio-economic strata in Kerala.

c. To study how the employment generation programs (MGNREGS) contributed to the nutritional status of children aged 6 to 60 months from poor socio-economic strata in Kerala.

\section{Methods/Design}

\subsection{Conceptual framework}

The theoretical framework adopted for this study is the eco-social theory proposed by Nancy Krieger in 1994. This Eco-social theory is a multi-level theory, which addresses who and what drives the unequal distribution of health in the society from micro to macro levels of one's life (Krieger, 2001, 2012). Since the present study attempts to explain the undernutrition among children from multiple levels, the eco-social theory provides a concrete basis to build further on the issue. The conceptual framework for the study is developed based on eco-social theory (see Figure 1).

Figure 1. Conceptual framework on social, economic and political dimensions of child nutrition.

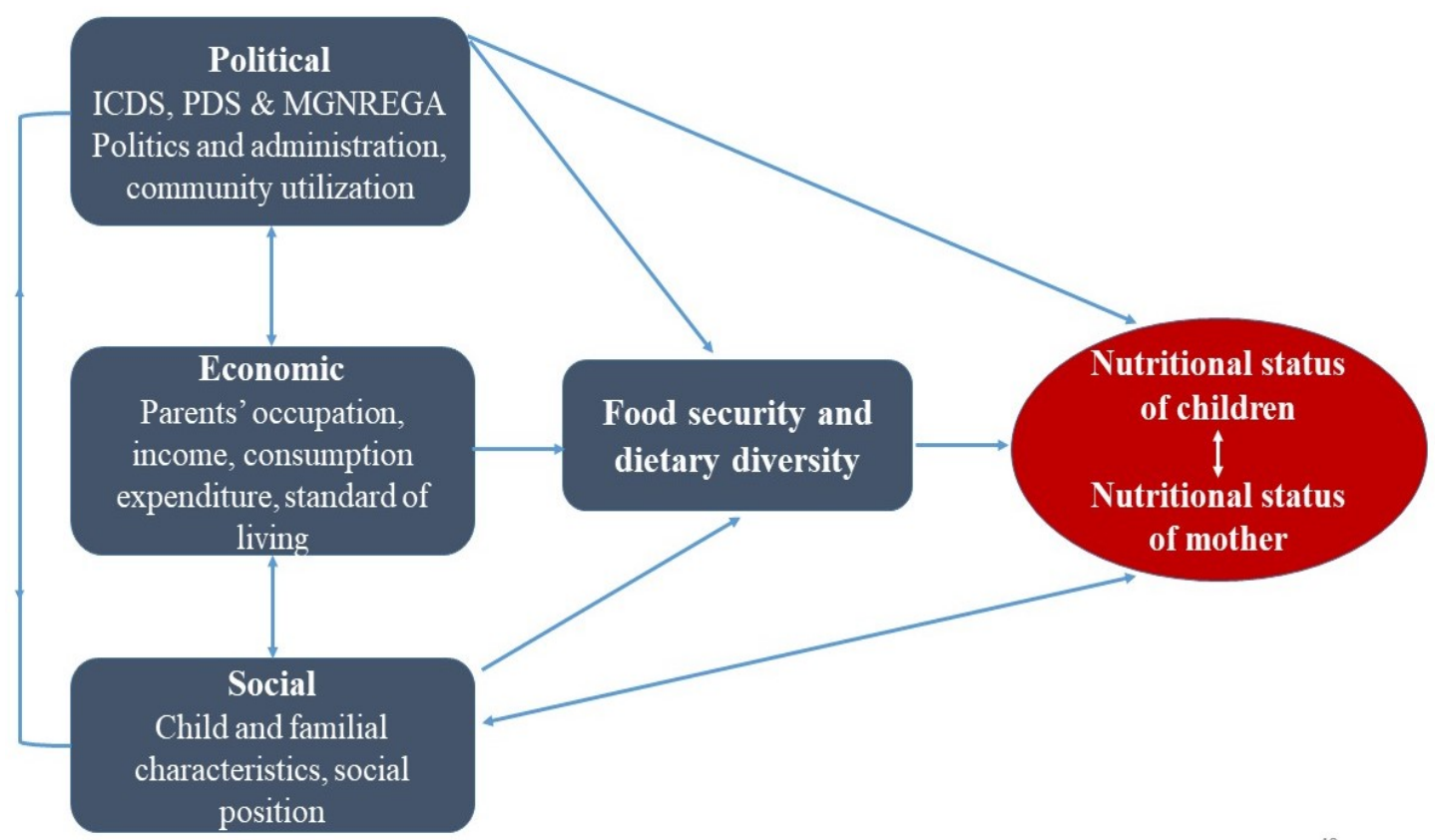

Social Science Protocols, February 2021, 1-12. 


\subsection{Study type/design}

The present study uses explanatory design. It attempts to explain the phenomenon of 'undernutrition among children' in Kerala. The study will use a multitude of methods, quantitative and qualitative data collection at a primary level along with the collection of secondary data to answer the research questions. There are three phases in the quantitative component of primary data collection. The first phase comprises a pilot study for validating tools. The second phase includes sample selection with a screening in order to select children aged 6 to 60 months belonging to low socio-economic strata, and finally the primary data collection phase. The qualitative phase includes in-depth interviews with key informants (listed in section 2.3) at the community and state levels including service providers, community representatives and political representatives.

\subsection{Study setting}

Kerala, India

\subsection{Participants and sampling}

\section{Quantitative phase}

The study looks into the role of ICDS, PDS and MGNREGS on the nutritional status of children aged 6 to 60 months. To achieve this, the study requires two groups for comparison: utilizers and non-utilizers. ICDS is the direct and the most studied intervention concerning the nutritional status of children. The targeted beneficiaries of ICDS are children belonging to zero to 72 months of age, pregnant women and lactating mothers. However, they (parents in case of children) have an option to opt-in or opt-out for availing the services including supplementary nutrition. In the present study, children of age 6-60 months, who avail the services specifically supplementary nutrition, will be included as the utilizers and those who do not avail services will be included as the non-utilizers. The difference in nutritional status between ICDS utilizers and non-utilizers was not reported from Kerala. Hence, a study from Utter Pradesh was considered for estimating the sample size for the present study.

The formula for calculating sample size in studies that compares two proportions is given below.

$$
n=\frac{P_{1}\left(1-P_{1}\right)+P_{2}\left(1-P_{2}\right)}{\left(P_{1}-P_{2}\right)^{2}} \times C p
$$

Where $\mathrm{n}=$ Sample size, $\mathrm{P} 1=$ Proportion of the event in Group 1, P2 = Proportion of the event in Group 2, Cp = Critical Value of $80 \%$ Power at 95\% confidence limits (Le, 2003; Pandis, 2012).

The prevalence of wasting among ICDS utilizers and non-utilizers was $12.9 \%$ and $22.6 \%$ respectively in a cross-sectional study conducted among 3-6 years old children in Uttar Pradesh (Pandey, Awasthi, Srivastava, Nigam, \& Srivastava, 2011). The study could find a significant difference with a sample size of 350. Considering this prevalence difference of $9.7 \%$ along with an assumed power of $80 \%$, the sample size was 488 . With an assumed nonresponse rate of $20 \%$ in total, the final sample size is 585.6 , rounded to 600 . For the study, 200 children and their mothers will be approached from each district.

Multi-stage sampling is planned for selecting participants for the survey. Three out of fourteen districts in Kerala are selected based on the prevalence of wasting reported by the NFHS-4. The 14 districts were categorized into three as follows: 1) districts with prevalence of wasting $<=10.0$ per cent (Kasaragod), 2) prevalence of wasting 10.1-20 per cent (Kannur, Kozhikode, Palakkad, Pathanamthitta, Thiruvananthapuram, Alappuzha, Ernakulum, Kollam, Social Science Protocols, February 2021, 1-12. 
Kottayam, Thrissur) and 3) prevalence of wasting $>20.0 \%$ (Idukki, Wayanad and Malappuram) (IIPS \& ICF, 2017b). One district from each of these categories is selected. It includes Kasaragod, Thiruvananthapuram and Idukki districts. These districts represent the northern, southern and central parts of Kerala, and thus provide a better representation of the state enhancing the generalizability of study findings. Further sampling is employed within each district. The sampling strategy planned in Idukki district is presented in Figure 2 and the same is applied in other two districts.

At the level of Anganwadi Centre (AWC) (community centres under ICDS to provide the services) a screening will be done to identify children belonging to low socio-economic strata. Children from historically disadvantaged sections of the society such as Dalit, tribal and fishing communities; those belonging to households having Antyodaya Anna Yojana (AAY) or Priority Household (PH) ration card holders (Department Food and Civil Supplies, 2018), and those from households where the primary income earner is a female/physically or mentally disabled person/elderly (above 60 years of age)/a teenager less than 22 years of age; and children of wage labourers in unorganized sectors will be included into the study without further scrutiny. Others will be included after asking 10 questions based on possession of various assets at the household. Children from households that do not possess of any of the items will be included in the study.

Further, ICDS utilizers and non-utilizers among these children will be selected by stratified random sampling. Children who were too sick to take measurements at the time of data collection, those who were differently abled or with congenital disorders (children with autism or cerebral palsy), and children of mothers who were not in a physical or mental stability to provide information about the children were not selected.

Figure 2. Plan of multistage random sampling for the cross-sectional survey.

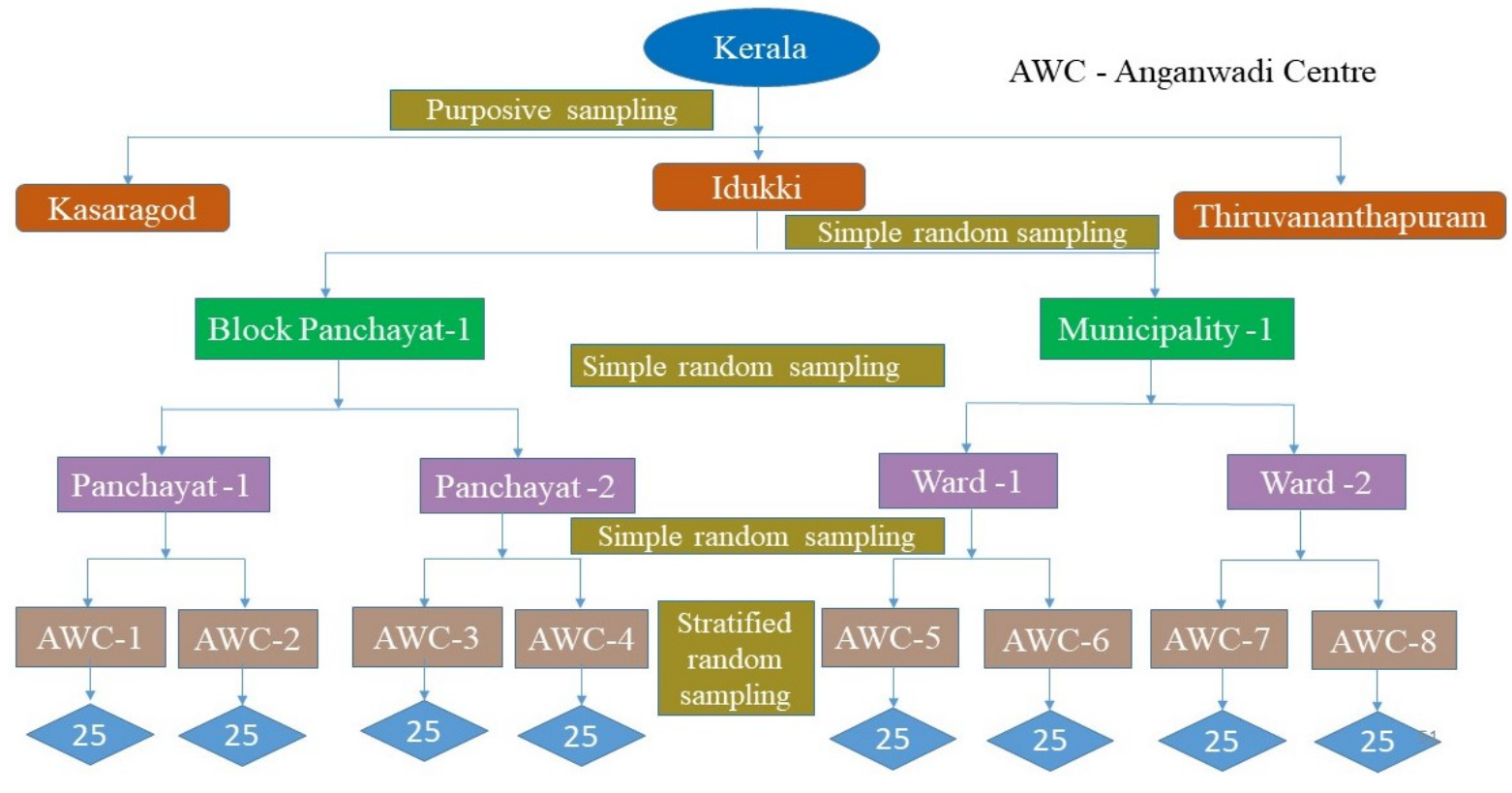

\section{Qualitative phase}

The qualitative phase includes in-depth interviews with key informants such as representatives of community organizations (religious leaders, representatives of self-help groups), service providers at local, block and district levels (Anganwadi workers, ICDS supervisors, PDS providers, MGNREGS administrators and policymakers (Local Self 
Government Institution-LSGI members, Members of Legislative Assembly (MLAs), Members of Parliament (MPs) serving the selected study areas. Participants who consent either verbally or on written consent form to participate in the study will be approached after taking prior appointments.

\section{Secondary Data}

The secondary data includes relevant documents such as National Food Security Act (NFSA, 2013), NFSA State Rules of Kerala (2018), ICDS Manual for district-level functionaries (2017), Legislative Assembly questions asked by MLAs in Kerala. In addition, two analyses were done using the first and the second India Human Development Survey (IHDS) data (Jayalakshmi \& Kannan, 2019a, 2019b).

\subsection{Data collection}

The study will use multiple tools for data collection including household survey questionnaire, interview schedule for mothers, interview guide for key informants, checklist for analyzing documents and field diary. In addition, anthropometric measurements of children and their mothers will be taken. The weight and height measured using the standardized instruments such as SECA 813 electronic weighing scale and SECA 213 portable stadiometer. For children up to the age of 18 months, an infantometer (NET WSO25) manufactured by Narang Medical limited for clinical purpose will be used to measure the length after calibration with SECA 201 non-elastic measuring tape. The procedure for taking measurements will strictly follow the WHO guidelines (WHO, 1995). Two measurements will be taken for each with a three minutes' interval. The height and weight measurements will be compared with the z-scores of the reference population given by the WHO (WHO, 2008).

The tools developed are based on thorough and comprehensive literature review. Household-level tools will be piloted among 15-30 potential participants. These mothers and children will become the study participants of the main study if they meet the screening criteria.

\subsection{Variables and data sources}

The specific dependent variable of the study is nutritional status of the child in terms of stunting, underweight and wasting among children. The independent variables identified through rigorous literature review are listed in Table 2.

\subsection{Data analysis}

Primary data on quantitative and qualitative methods to be analyzed separately as follows. Quantitative data will be analyzed for understanding the prevalence of undernutrition in terms of stunting, underweight and wasting. Further, bivariate and multivariate analysis will be done to find out how the different factors under social, economic and political dimensions is associated with undernutrition among children in the study. Thematic analysis will be used for qualitative data. Content analysis of relevant secondary data will be done. The integration of the findings may be done in the discussion as required.

\subsection{Strengths of the study}

The nutritional status of children in Kerala is often overlooked under the notion of 'model development'. This study will reveal the status of the same in the state and untangle the 
causes too through a comprehensive approach to understand factors playing at multiple levels.

\subsection{Limitations of the study}

The findings may not be generalizable to other states in India as they considerably differ from Kerala on various aspects. However, assuming that they are also facing a similar developmental transition in the present time, results may be generalised.

\subsection{Ethical considerations}

The informed consent process is in confirmation with the Ethics Committee requirements. Mothers' consent will be taken to include their children into the study. Privacy and confidentiality of all participants will be ensured through masking the identifiers throughout the study. Principal investigator will have the sole responsibility of safekeeping the data. The participant information sheet will be provided with the contact details of principal investigator for any clarifications. Utmost care will be taken to ensure comfort of all participants during the data collection process.

\section{Discussion}

As described in the background, the context of Kerala is different from many other states in India, especially in terms of development. Therefore, it is important to consider these contextual differences while intervening to address the problem of undernutrition among children.

The study will critically look into the developmental scenario in Kerala and its effects on the nutritional status of children. It will reveal the gaps in the present system to address undernutrition among children. These results will have implications at individual, service provision and policy levels. The gaps identified will serve as the evidence for reforming the present system in a way that suits the social, cultural and developmental environment of Kerala.

\section{Declarations}

Acknowledgement: We thank the Technical Advisory Committee members and Doctoral. Advisory Committee Members at Sree Chitra Tirunal Institute for Medical Sciences and Technology, Trivandrum, India for their valuable comments and suggestions.

\section{Funding: Nil}

Conflicts of interest: The authors declare that there are no competing interests.

Ethics approval and consent to participate: The Institutional Ethics Committee of Sree Chitra Tirunal Institute for Medical Sciences and Technology, Trivandrum, Kerala, India approved the study (Reference number IEC/1272, dated September 6, 2018). All participants will be given written informed consent before administering the tools.

\section{References}

Anand, S., \& Sen, A. (2000). Human development and economic sustainability. World Development, 28(12), 2029-2049. https://doi.org/10.1016/S0305-750X(00)00071-1 
Birhanu, M. M. (2015). Systematic reviews of prevalence and associated factors of under five malnutrition in Ethiopia: Finding the evidence. International Journal of Nutrition and Food Sciences, 4(4), 459.

http://article.sciencepg.net/html/10.11648.j.ijnfs.20150404.17.html

Black, R. E., Victora, C. G., Walker, S. P., Bhutta, Z. A., Christian, P., De Onis, M., ... Martorell, R. (2013). Maternal and child undernutrition and overweight in low-income and middle-income countries. The Lancet, 382(9890), 427-451. https://doi.org/10.1016/S0140-6736(13)60937-X

Chaturvedi, S., Ramji, S., Arora, N. K., Rewal, S., Dasgupta, R., \& Deshmukh, V. (2016). Time-constrained mother and expanding market: Emerging model of under-nutrition in India. BMC Public Health, 16, 632. https://doi.org/10.1186/s12889-016-3189-4

Department Food and Civil Supplies. (2018). NFSA State Rule. Government of Kerala. https://civilsupplieskerala.gov.in/index.php/cards

Devika, J. (2010). Egalitarian developmentalism, communist mobilization, and the question of caste in Kerala state, India. The Journal of Asian Studies, 69(3), 799-820. https://doi.org/10.1017/s0021911810001506

Fenske, N., Burns, J., Hothorn, T., \& Rehfuess, E. A. (2013). Understanding child stunting in India: A comprehensive analysis of socio-economic, nutritional and environmental determinants using additive quantile regression. PLOS ONE, 8(11), e78692. https://doi.org/10.1371/journal.pone.0078692

George, P. S., \& Chattopadhyay, S. (2001). Population and land use in Kerala. In Growing populations, changing landscapes: Studies from India, China, and the United States. National Academies Press. https://doi.org/10.17226/10144

International Institute for Population Sciences \& Inner City Fund. (2017a). National Family Health survey (NFHS-4), 2015-16: India. http://rchiips.org/NFHS/NFHS4Reports/India.pdf

International Institute for Population Sciences \& Inner City Fund. (2017b). National Family Health Survey (NFHS-4), India, 2015-16: Kerala. http://rchiips.org/NFHS/NFHS4Reports/Kerala.pdf

Jayalakshmi, R., \& Kannan, S. (2019a). The catch-up growth in stunted children: Analysis of first and second India human development survey data. Indian Journal of Community Medicine, 44(3), 199. https://doi.org/10.4103/ijcm.IJCM_127_18

Jayalakshmi, R., \& Kannan, S. (2019b). The double burden of malnutrition: An assessment of 'stunted child and overweight/obese mother (SCOWT) pairs' in Kerala households. Journal of Public Health Policy, 40(3), 342-350. https://doi.org/10.1057/s41271-01900172-7

Kanter, R., Walls, H. L., Tak, M., Roberts, F., \& Waage, J. (2015). A conceptual framework for understanding the impacts of agriculture and food system policies on nutrition and health. Food Security, 7(4), 767-777. https://doi.org/10.1007/s12571-015-0473-6

Kerala State Planning Board. (2020). The economic review 2019 https://spb.kerala.gov.in/economic-review/ER2019/index.php

Krieger, N. (2001). Theories for social epidemiology in the 21st century: An ecosocial perspective. International Journal of Epidemiology, 30(4), 668-677. https://doi.org/10.1093/ije/30.4.668

Krieger, N. (2012). Methods for the scientific study of discrimination and health: An ecosocial approach. American Journal of Public Health, 102(5), 936-944. https://doi.org/10.2105/AJPH.2011.300544

Le, C. T. (2003). Introductory biostatistics. Wiley-Interscience. 
Nisbett, N., Gillespie, S., Haddad, L., \& Harris, J. (2014). Why worry about the politics of childhood undernutrition? World Development, 64, 420-433. https://doi.org/10.1016/j.worlddev.2014.06.018

Pandey, V., Awasthi, S., Srivastava, V., Nigam, A., \& Srivastava, P. (2011). Study of nutritional status of children attending ICDS services in Lucknow. Indian Journal of Preventive and Social Medicine, 42(2), 142-144. https://drive.google.com/file/d/1diEnwGqXxn3Mtt5ZEmlxfmbgBLYjNcXd/view

Pandis, N. (2012). Sample calculations for comparing proportions. American Journal of Orthodontics and Dentofacial Orthopedics, 141(5), 666-667. https://doi.org/10.1016/j.ajodo.2012.02.001

Parayil, G. (1996). The "Kerala model" of development: Development and sustainability in the third world. Third World Quarterly, 17(5), 941-958. https://doi.org/10.1080/01436599615191

Philip, R., Vijayakumar, K., Indu, P., Shrinivasa, B., Sreelal, T., \& Balaji, J. (2015). Prevalence of undernutrition among tribal preschool children in Wayanad district of Kerala. International Journal of Advanced Medical and Health Research, 2(1), 33. https://doi.org/10.4103/2349-4220.159135

Planning Commission. (2010). Addressing India's nutrition challenges: Report of the multistakeholder retreat. Government of India.

Prabhakaran, A. O., Ekbal, B., Jayasree A K, Urmila, K., Krishnanunni, \& Renjith, P. (2013). Report on health status of tribal in Attapadi. Pariyaram Medical College. http://rgdoi.net/10.13140/RG.2.1.2616.8400

Ramachandran, V. K. (2000). Human development achievements in an Indian state: A case study of Kerala. In D. Ghai (Eds.), Social Development and Public Policy (pp. 46-102). Palgrave Macmillan. https://doi.org/10.1057/9780230374232_2

Sabharwal, S. N. (2011). Caste, religion and malnutrition linkages. Economic and Political Weekly, xlvi(50), 16-18. https://www.epw.in/journal/2011/50/commentary/castereligion-and-malnutritionlinkages.html?0=ip_login_no_cache\%3D513a22bfe5c2171ebac76173d51d18e0

Sajithamohan, M., \& Arunachalam, P. (2018). A comparative assessment of nutritional status between tribal and non-tribal under five children in Malappuram district, Kerala. International Journal of Advanced Research, 6(3), 1436-1440. https://doi.org/10.21474/IJAR01/6817

United Nations Children's Fund, World Health Organization \& World Bank. (2019). Levels and trends in child malnutrition: Key findings of the 2019 edition of the joint child malnutrition estimates. Retrieved from https://www.who.int/nutgrowthdb/jme_brochoure2017.pdf

United Nations Children's Fund. (2019). Children, food and nutrition: Growing well in a changing world. New York. https://www.unicef.org/reports/state-of-worlds-children2019

United Nations Children's Fund. (2015). UNICEF's approach to scaling up nutrition for mothers and their children [Discussion Paper]. Programme Division. https://www.unicef.org/nutrition/files/Unicef_Nutrition_Strategy.pdf

United States Dietary Guidelines Advisory Committee. (2010). Dietary Guidelines for Americans, 2010 (No.232). U.S. Department of Health and Human Services, U.S. Department of Agriculture. https://health.gov/sites/default/files/202001/DietaryGuidelines2010.pdf

Van de Poel, E., Hosseinpoor, A. R., Speybroeck, N., Van Ourti, T., \& Vega, J. (2008). Socioeconomic inequality in malnutrition in developing countries. Bulletin of the World Health Organization, 86(4), 282-291. https://doi.org/10.2471/BLT.07.044800

Social Science Protocols, February 2021, 1-12.

http://dx.doi.org/10.7565/ssp.v4.5284 
Wondimagegn, Z. T. (2014). Magnitude and Determinants of Stunting Among Children in Africa: A Systematic Review. Current Research in Nutrition and Food Science Journal, 2(2), 88-93. http://dx.doi.org/10.12944/CRNFSJ.2.2.05

World Bank. (2017). Kerala - Poverty, growth, and inequality. Retrieved http://documents.worldbank.org/curated/en/714181504169813713/pdf/119248-BRIP157572-Kerala-Poverty.pdf

World Bank. (2017). Kerala: Social Inclusion. Retrieved from http://documents.worldbank.org/curated/en/947421504170192386/pdf/119249-BRIP157572-Kerala-Social.pdf

World Health Organization. (1995). Physical status: The use and interpretation of anthropometry: Report of a WHO Expert Committee. Geneva. https://apps.who.int/iris/bitstream/handle/10665/37003/WHO_TRS_854.pdf;jsessionid= 833C3A1C3F7041CC1B9155572E6F2CD6? sequence=1

World Health Organization. (2008). Training course on child growth assessment: Interpreting growth indicators. Geneva. https://www.who.int/childgrowth/training/module_c_interpreting_indicators.pdf

World Health Organization. (2010). Nutrition Landscape Information System (NLIS) country profile indicators: interpretation guide. Geneva. https://www.who.int/nutrition/nlis_interpretation_guide.pdf

World Health Organization. (2020). Children: improving survival and well-being. Retrieved from https://www.who.int/en/news-room/fact-sheets/detail/children-reducing-mortality 